\section{Aeroelasticity Surveyed}

THe second Lanchester Memorial Lecture, given on November 20 before the Royal Aeronautical Society by Prof. A. R. Collar, of the University of Bristol, was entitled "Aeroelasticity-Retrospect and Prospect". Prof. Collar began with a historical survey, mentioning the investigation of flutter made by Lanchester in 1916 before turning to the pioneer work of Duncan and Frazer, who published a paper on "The Flutter of Aeroplane Wings" (1928), which is still a standard reference. Problems of flutter, wing and tailplane divergence, fuselage bending and control reversal multiplied with the advent of the monoplane and during the intense development period of the Second World War. Prof. Collar reiterated his earlier suggestion that by treating the dynamics of a deformable aircraft, all aeroelastic problems could be solved, and added the ray of hope that electronic computers may soon be able to turn a theoretical possibility into a practical reality. He thought that the important recent developments of flight simulators, ground resonance tests and elastic wind-tunnel models, together with greatly increased industrial interest in aeroelastic problems, would ensure fewer accidents in the future. Prof. Collar stressed the need for a better understanding of the effects of damping, which should suppress but often only aggravates flutter. He also wanted to seo better methods for estimating air forces. The future problems of very high-speed flight will present kinetic heating effects liable to distort the slender wing sections to a degree not previously experienced. A powerful analytical treatment will be needed.

\section{Tokyo Observatory Polar Tube}

THE determination of the position of the celestial pole is of interest in connexion with the construction of star catalogues, the measurement of the precise values of the astronomical constants of precession, nutation and aberration and the movement of the pole due to geophysical disturbances. The meridian circle has been employed in the past for this kind of work, but the installation of a fixed telescope pointing to the pole has certain advantages, including the use of the same star-field at each observation and the simplified structure leading to less complicated instrumental errors. This idea was first put into practice at the Poulkovo Observatory near Loningrad, and a similar telescope, which they named a polar tube, has been constructed at the Tokyo Observatory by N. Sekiguchi and J. Matsumoto (Annals Tokyo Astro. Obs., 5, 131; 1957; Pub. Astro. Soc. Japan, 9, 116; 1957). The objective of diameter $20 \mathrm{~cm}$. has a focal length of $269 \mathrm{~cm}$. The photographic plate is rotated about the optical axis at the sidereal rate so that stars are photographed as point images. An almost plane-parallel glass plate in front of the objective has at its centre a small aluminized reflecting surface, inclined to the plate at such an angle as to cause light from the optical axis of the polar tube to be reflected vertically downwards into a mercury basin. A pencil of light is led into the tube. Some is reflected from the glass plate to form an image on the photographic plate. Some is reflected from the reflector, mercury and reflector again and forms a second image, shifted slightly from the first image. Changes of position of the two images give information on the motion of the plate relative to the objective. The plate is exposed twice, for about five minutes on each occasion, at an interval of five or six hours. Ten tenth-magnitude stars are used.
The common point of the two star-fields photographed is, after certain corrections have been applied, the position of the celestial pole at that date. Preliminary observations suggest a probable error of 0.3 second of arc for one observation.

\section{Transformation Characteristics of Direct-hardening Nickel Alloy Steels}

A MONOGRAPH entitled "Transformation Characteristics of Direct Hardening Nickel Alloy Steels", recently issued by the Mond Nickel Co., Ltd. (pp. 91 . London, 1958), is a revised and enlarged edition of the "Atlas of Isothermal Transformation Diagrams of Nickel Steels" previously published. The information contained in the sixty pages of text and twentyfive pages of diagrams is excellently presented, and the bibliography of eighty-six references adds further to the utility of this little book. In addition to the isothermal diagrams and data on end-quench harden. ability previously reproduced, the revised publication incorporates supplementary information in the form of continuous-cooling transformation diagrams. This is of value, since the difficulty of applying isothermal data to practical heat-treatments lies in the fact that it is not possible to assess reliably from isothermal diagrams the extent and type of transformations which may occur during continuous cooling. The diagrams shown are for sections from 1 in. to 6 in. in diameter, quenched in oil. Both smaller and much larger sections are of course used, and other cooling media, but this information will probably prove the most generally useful to the engineer in the treatment and application of steels containing nickel.

\section{Charles Leopold Meyer Award for 1958}

The Société de Chimie Biologiquo has awarded the Charles Leopold Meyer Award for 1958 jointly to Dr. François Gros of the Institut Pasteur, Paris, and to Prof. Jean Turchini, of the faculty of medicine, Montpellier. This annual award of 500,000 franes is made for research on the nucleic acids. Dr. Gros has worked on the mechanism of action of various antibiotics on bacteria ; in particular he has studied how antibiotics modify the degradation and re. constitution of the nucleic acids in the cell. Prof. Turchini has conducted a number of investigations over a period of years, partly in collaboration with Prof. P. Castel and Dr. K. van Kien, using specifie staining techniques to localize certain nucleic acids within the cell and to follow their evolution under the microscope.

\section{Paul Instrument Fund Awards}

Awards of grants by the Paul Instrument Funcl Committee have been made as follows: $£ 3,835$ to Prof. A. L. Cullen, professor of electrical engineering in the University of Sheffield, for the construction of a detector in which radiation pressure is used to convert a microwave signal to an audio or intermediate frequency; $£ 545$ to Dr. R. Feinberg, of the Department of Electrical Engineering, Manchester College of Science and Technology, for the construction of an instrument for measuring the intensity of soft X-radiation generated in experimental highvoltage valves; and $£ 1,000$ to Mr. R. M. Sillitto, lecturer in natural philosophy, University of Edinburgh, for the development of a thirty-channel pulse-height analyser, using transistors in place of thermionic valves.

The Paul Instrument Fund Committee, composed of representatives of the Royal Society, the Physical 